University of Nebraska - Lincoln

DigitalCommons@University of Nebraska - Lincoln

January 1995

\title{
Effects of track structure and cell inactivation on the calculation of heavy ion mutation rates in mammalian cells
}

Francis A. Cucinotta

NASA Langley Research Center, Hampton, VA, francis.cucinotta@unlv.edu

J. W. Wilson

NASA Langley Research Center, Hampton, VA

M. R. Shavers

Texas A\&M University, College Station, $T X$

Robert Katz

University of Nebraska-Lincoln, rkatz2@unl.edu

Follow this and additional works at: https://digitalcommons.unl.edu/physicskatz

Part of the Physics Commons

Cucinotta, Francis A.; Wilson, J. W.; Shavers, M. R.; and Katz, Robert, "Effects of track structure and cell inactivation on the calculation of heavy ion mutation rates in mammalian cells" (1995). Robert Katz Publications. 56.

https://digitalcommons.unl.edu/physicskatz/56

This Article is brought to you for free and open access by the Research Papers in Physics and Astronomy at DigitalCommons@University of Nebraska - Lincoln. It has been accepted for inclusion in Robert Katz Publications by an authorized administrator of DigitalCommons@University of Nebraska - Lincoln. 


\title{
Effects of track structure and cell inactivation on the calculation of heavy ion mutation rates in mammalian cells
}

\author{
F. A. CUCINOTTA, ${ }^{*}+$ IP J. W. WILSON, $†$ M. R. SHAVERS $\S$ and R. KATZף \\ (Received 26 May 1995; revision received 19 January 1996; accepted 25 January 1996)
}

\begin{abstract}
It has long been suggested that inactivation severely effects the probability of mutation by heavy ions in mammalian cells. Heavy ions have observed cross sections of inactivation that approach and sometimes exceed the geometric size of the cell nucleus in mammalian cells. In the track structure model of Katz the inactivation cross section is found by summing an inactivation probability over all impact parameters from the ion to the sensitive sites within the cell nucleus. The inactivation probability is evaluated using the dose-response of the system to $\gamma$-rays and the radial dose of the ions and may be equal to unity at small impact parameters for some ions. We show how the effects of inactivation may be taken into account in the evaluation of the mutation cross sections from heavy ions in the track structure model through correlation of sites for gene mutation and cell inactivation. The model is fit to available data for HPRT mutations in Chinese hamster cells and good agreement is found. The resulting calculations qualitatively show that mutation cross sections for heavy ions display minima at velocities where inactivation cross sections display maxima. Also, calculations show the high probability of mutation by relativistic heavy ions due to the radial extension of ions track from $\delta$-rays in agreement with the microlesion concept. The effects of inactivation on mutations rates make it very unlikely that a single parameter such as LET or $Z^{* 2} / \beta^{2}$ can be used to specify radiation quality for heavy ion bombardment.
\end{abstract}

\section{Introduction}

The level of biological injury to be expected from galactic cosmic rays (GCR) during prolonged manned spaceflight is difficult to estimate because of the lack of human data from exposures to high charge and energy (HZE) particles. Experimental studies for estimating the risk from long-term

*Author for correspondence.

WASA Langley Research Center, Hampton, VA 23681OOOI, USA.

tThe Johns Hopkins School of Medicine, Division of Radiation Oncology, Baltimore, MD 21205, USA.

Texas A\&M University, College Station, TX 77843, USA.

University of Nebraska, Lincoln, NE 68588, USA.

Present address: On Sabbatical Leave at The oncology Center, Johns Hopkins School of Medicine, Baltimore, MD, 21287, USA
GCR exposures include track segment irradiations with HZE particles in which animals or cell cultures are used. The most useful end points for such studies with animals are cancer induction and mortality. Cellular studies using cytotoxicity as an end point are useful for providing estimates of relative biological effectiveness (RBE), whereas the stochastic end points of mutagenesis or neoplastic transformation provide RBEs for more important end points and additional information on the late effects that may be useful in extrapolations of the level of risk for man and on the underlying mechanisms of damage from HZE particles.

An experimental assay has been developed for studies of mutations at the hypoxanthine guanine phosphoribosyl transferase (HPRT) locus in mammalian cell cultures. The HPRT gene is located on the $X$ chromosome, and the mutation of this gene is related to DNA damage. The HPRT locus is a well-studied system for considering the expression of large deletions or rearrangements (Cox and Masson 1979, NCRP 1990). This assay system has been used by several groups with a variety of light and heavy ion types in several cell lines (Cox and Masson 1979, Thacker et al. 1979, Kronenberg and Little 1989, Kranert et al. 1990). One of the shortcomings of this assay system is that the chromosome involved is necessary for cell replication, which results in the loss of potential mutants. Human-hamster hybrid systems are being used to obtain higher mutation rates in other studies (NCRP 1990). Also, measurements (Kronenberg and Little 1990) of mutations to trifluorothymidine resistance locus indicate slow-growth mutants that are not typical of the HPRT mutants, an indication that some variability exists in mutations at specific genetic loci in human cells.

In describing mutagenesis from heavy ions, the question arises of whether there will be any mutations observed at all if single tracks of heavy ions kill the cell due to the large energy they deposit in the cell nucleus. Goodhead et al. (1980) and 
Kranert et al. (1990) discussed this problem, noting that for heavy ions the inactivation cross section is generally smaller than the nuclear area for mammalian cells and that track structure effects should be considered in order to understand the role of inactivation of heavy ion mutagenesis. Studies by Lett et al. (1989) with repair deficient LS1784 S/S cells show that inactivation cross sections may exceed the geometric cross section in some cases. In this paper we use the track structure model of Katz et al. $(1971,1985)$ in order to evaluate the mutation cross section for ion bombardment of Chinese hamster fibroblasts (V79). We show that the mutation cross section for ions throughout the periodic table can be described by the track model when inactivation effects are accounted for in the model. In effect, we will have a qualitative model of what has long been suggested (Grahn 1973, Todd 1983, Kiefer 1993) of heavy ion mutagenesis, including the concept of a microlesion where mutated cells surround a core of inactivated ones.

The action cross section was first described by Butts and Katz (1967) using the spatial distribution of energy about an ion to map the response of low linear energy transfer (LET) irradiations such as $\gamma$-rays or electrons to that of an ion. An inactivation probability as a function of impact parameter or radial distance about the ion can be described in this manner using the ions' radial dose and the $\gamma$-ray response function. For a finite target size, the radial dose is averaged over the target volume assumed to be a short cylinder of radius $a_{0}$. Several experiments have now been performed (Facius et al. 1983, Weissbrod et al. 1992) to measure the inactivation probability $P(t)$ for ion bombardment of Bacillus subtilis spores. These measurements appear to indicate that the probability is not unity for small impact parameters for uranium bombardments seeming to contradict the predictions of the track structure model. Recently, calculations for the inactivation probability of spores (Cucinotta et al. 1995) hate shown that if the sensitive targets in the spores are allowed to be displaced from the center of the spore volume, a good representation of these measurements is provided by the track model and a unit probability for small impact parameters is not ruled out. Recent measurements of the DNA content in yeast cells by Kost and Kiefer (1993) also support the assertion that the impact parameters in the inactivation measurements are likely to be displaced from the sensitive site(s) for inactivation.
The experimental data for the dose-response for mutations from $\gamma$-rays in mammalian cells is severely limited. Data below doses of $0.5 \mathrm{~Gy}$ are difficult to obtain due to poor statistics. Data above doses $10 \mathrm{~Gy}$ are also difficult to obtain due to the high inactivation rates leading to poor recovery of mutants. The track structure model relies on extrapolating effects of low LET irradiations at high dose-rate to that of ions using the low-LET dose-response. The extrapolation becomes difficult due to the limited range of data for the dose-response. The track model employs a multitarget or multihit model for the functional form of the dose-response. Wilson $e t$ al. (1993) have developed a linear repair/misrepair kinetics model for multiple lesion formation appropriate for the case of mutation in competition with inactivation. We consider both the multitarget and linear kinetics model for the low LET dose-response model in this paper.

Our accounting of inactivation effects on heavy ion mutation cross sections relies on the assumption that the sensitive sites for inactivation may be displaced from that of mutation. We average the displacement distance over the nuclear volume for the V79 cells; this leads to a good representation of the existing measurements of mutation cross sections in V79 cells. Parameters for cell inactivation are fixed in the model from inactivation data. The resulting model shows, in agreement with the data, that the mutation cross section for very heavy ions plotted as a function of ion energy is a minimum when the inactivation cross section is a maximum. Also, we show that the effects of inactivation on mutations from light ions is small. In the remainder of this paper we first describe the calculation of the mutation cross section in the track model when inactivation is considered. The model is then fit to the existing data for V79 cell HPRT mutations and the mutation probability as a function of ion charge and energy, is discussed.

\section{Inactivation and mutation cross sections in track model}

In order to introduce the effects of survival probability on the evaluation of mutation cross sections in the track model we first review the evaluation of the inactivation cross section (Butts and Katz 1967, Katz et al. 1971). The doseresponse of the system to low LET irradiations 
for the endpoint of inactivation (loss of colonyforming ability) is assumed to be known and represented by the probability function $P_{\mathrm{I}}(D)$, where $D$ is the absorbed dose in Gy. Track structure is understood by finding the spatial distribution of local dose about the path of an ion, as deposited in the sensitive volume elements of the cell. For calculations, the average radial dose as a function of the radial distance $t$ is a short cylinder of radius $a_{0_{\mathrm{I}}}$ is used to denote $\bar{D}_{\mathrm{I}}(t)$. The inactivation cross section of a single putative target is found by integrating the probability for inactivation evaluated with the average radial dose in $a_{0_{1}}$ over all radial distance, as (Katz et al. 1971)

$$
\sigma_{\mathrm{I}}=\int_{0}^{T_{\max }} 2 \pi t P_{\mathrm{I}}\left(\bar{D}_{\mathrm{I}}(t)\right) \mathrm{d} t
$$

where $T_{\max }$ is the maximum range of the $\delta$-rays, often denoted the penumbra radius, which is a function of ion velocity $\beta$.

In implementing equation 1 for describing survival curves a multitarget model for low LET dose-response is often assumed where

$$
P_{\mathrm{I}}(D)=\left[1-\mathrm{e}^{-D / D_{0_{\mathrm{I}}}}\right]^{m},
$$

where $m$ is the target number and $D_{0_{1}}$ is the low LET characteristic dose, at which there is an average of one hit per target. The surviving fraction of cells after low LET irradiation at $D$ is $N / N_{0}=1-P_{\mathrm{I}}(D)$. The use of a multitarget model to fit the high dose-rate, low LET doseresponse is convenience in the calculations, and it can be shown (Cucinotta et al. 1995a) that other parametric models will work equally as well in predicting action cross sections for ions using equation 1.

The inactivation cross section evaluated from equation 1 plotted as a function of an ion's velocity is observed to reach a plateau at a about $1-1 \cdot 4 \pi a_{0_{\mathrm{I}}}^{2}$ marking the transition from the grain count regime to the track-width regime (Katz et al. 1971). The sensitive targets for the case of $m>1$ are contained in some volume represented by the cross sectional area $\sigma_{0_{\mathrm{I}}}$ which may be less than the total cross sectional area of the cell nucleus. The cross sections calculated by equation 1 are then multiplied by the ratio $\sigma_{0_{1}} / 1 \cdot 4 \pi_{0_{1}}^{2}$ when $m>1 . a_{0_{1}}$ and $\sigma_{0_{1}}$ are determined by fitting the model equations to the experimental data set using track segment irradiations. In the track model, the surviving fraction after irradiation with track segment ion bombardments is separated into intratrack and intertrack effects as

$$
\frac{N}{N_{0}}=\pi_{i} \times \pi_{\gamma},
$$

where the intratrack or ion-kill contribution is given by

$$
\pi_{i}=\mathrm{e}^{-\sigma_{i} F},
$$

where $F$ is the ion's fluence and the intertrack or $\gamma$-kill probability is

$$
\pi_{\gamma}=1-P_{\mathbf{I}}\left(D_{\gamma}\right)
$$

where $D_{\gamma}$ is the $\gamma$-kill dose given by

$$
D_{\gamma}=\left(1-\frac{\sigma_{\mathrm{I}}}{\sigma_{0_{\mathrm{I}}}}\right) F,
$$

with $D_{\gamma}=0$ for $\sigma_{\mathrm{I}}>\sigma_{0}$.

At sufficiently large fluence, the survival curves resulting from equation 1 to 6 display an exponential tail and the extrapolated cross section can be found as (Katz et al. 1971)

$$
\sigma_{\mathrm{ext}}=\sigma_{\mathrm{I}}+\left(1-\frac{\sigma_{\mathrm{I}}}{\sigma_{0_{\mathrm{I}}}}\right) \frac{L E T}{D_{0_{1}}} .
$$

The extrapolated cross section is also termed the final slope 'cross section' and is identical to $\sigma_{1}$ (the initial slope cross section) only when $\sigma_{1}>\sigma_{0_{1}}$.

In order to evaluate the mutation cross section in the track model the probability of survival of the cell must be considered in order for the mutation phenotype to be expressed. Unlike the target(s) for inactivation, the target(s) for gene

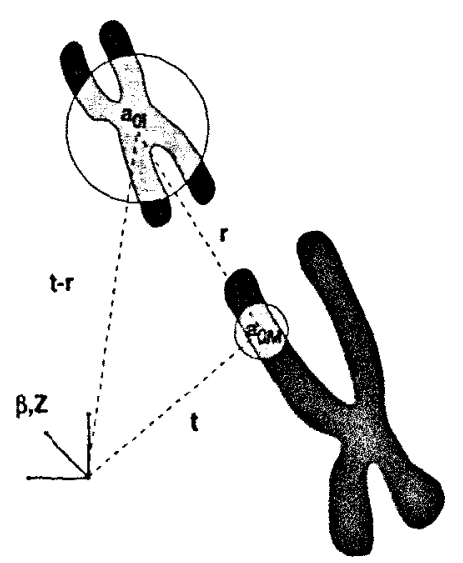

Figure 1. Schematic diagram of correlation of lesion sites for mutation and inactivation relative to path of ion of velocity $\beta$ and charge $Z$ at impact parameter $t$ from mutated gene with $r$ displacement of two sites. In calculations, $r$ is averaged over nuclear volume. 
mutation are well localized. For the HPRT mutation assay these are on the $X$ chromosome. We will assume that the targets for inactivation are located randomly in the cell nucleus (i.e. not chromosome specific). In Figure 1 we depict the sites for mutation and interactivation relative to the ion's path.

The cross section for mutation is evaluated by considering the dose-response probability per surviving cell for low LET induction of the mutation, denoted $P_{\mathrm{M}}$, multiplied by the doseresponse probability that the cell survives. The product of the mutation frequency per survivor, $P_{\mathrm{M}}$, times the survival probability, $1-P_{\mathrm{I}}$, represents the number of mutants observed. Track structure is introduced by correlating the location of these events within the cell nucleus. The cross section for production of observable mutants is then

$$
\begin{aligned}
\sigma_{\mathrm{M}}= & \int_{0}^{T_{\max }} 2 \pi t \mathrm{~d} t P_{\mathrm{M}}\left(\bar{D}_{\mathrm{M}}(t)\right) \frac{1}{V_{\mathrm{N}}} \\
& \times \int \mathrm{d} \vec{r}\left(1-P_{\mathrm{I}}\left(\bar{D}_{\mathrm{I}}(\vec{t}-\vec{r})\right)\right)
\end{aligned}
$$

where $V_{N}$ is the volume of the cell nucleus and the radial dose at the gene, $\vec{D}_{\mathrm{M}}$, is averaged over the mutation site of radius $a_{0 \mathrm{M}}$. The success of equation 8 , as shown in calculations below, is to correlate the spatial distributions of lesions for these endpoints. In calculations, $V_{N}$ is restricted to account for the finite size of $a_{0_{\mathrm{I}}}$ such that the displacement keeps the inactivation sites inside the nuclear volume. We expect the displacement distance then to be localized within about $3 \mu \mathrm{m}$ from the mutation site. For calculations, both a spherical and cylindrical geometry for the volume integral in equation 8 were considered with similar results found and therefore a spherical volume is assumed in the results discussed below. Many factors, such as the finite chromosome number, chromosome geometry, temporal position, etc., preclude any ab-initio correlation of the mutation and inactivation lesion sites. Its actual value for calculations is described below.

\section{Calculations of cross sections}

For calculations of cross sections the radial dose from secondary electrons based on the model of Kobetich and Katz (1968) is used. We have updated some of the physical inputs in this calculation (Cucinotta et al. 1995b), including the use of
Table 1. Cellular response parameters for V-79 cells

\begin{tabular}{lcccr}
\hline Endpoint & $m$ & $D_{0}(\mathrm{~Gy})$ & $\sigma_{0}\left(\mu \mathrm{m}^{2}\right)$ & $a_{0}(\mathrm{~nm})$ \\
\hline Inactivation & 3 & 1.82 & 42.8 & 820 \\
HPRT mutations & 2 & 950 & $6.5 \times 10^{-3}$ & 50 \\
\hline
\end{tabular}

the secondary electron spectrum from proton impact in water from Rudd (1989), a revised angular distribution ansatz, and the electron range-energy and stopping power formula from Tabata et al. (1972). Also, we have included a contribution for excitations to the radial dose model using the ansatz of Brandt and Ritchie (1974), normalized such that the summed contributions from excitations and $\delta$-rays (from modified Kobetich and Katz model) conserves the LET for each ion where

$$
L E T=2 \pi \int_{0}^{T_{\max }} t \mathrm{~d} t\left[D_{\delta}(t)+D_{\mathrm{exc}}(t)\right] .
$$

We have not considered the effects of nuclear stopping power which should become important at low energies $(<1 \mathrm{MeV} / \mathrm{u})$.

For survival, the $\mathrm{X}$-ray response parameters as well as the geometric parameters have been fitted by Katz et al. (1994) as listed in Table 1. For HPRT mutations the X-ray response in V79 cells has been measured by Kranert et al. (1989) for doses of 1-10 Gy. The multitarget model can be applied directly to the mutation frequency as shown by

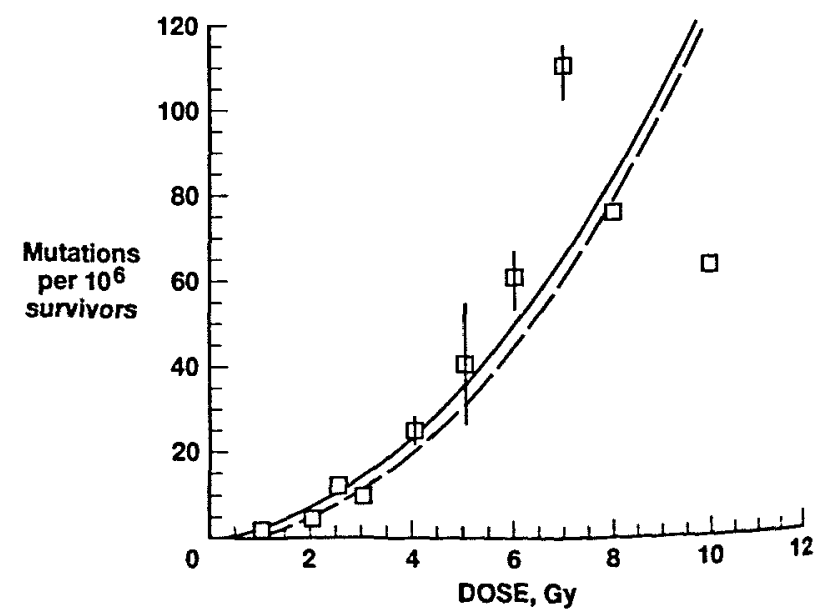

Figure 2. X-ray dose-response for the number of mutations per $10^{6}$ surviving cells for HPRT mutations in 179 cells. Data are from Kranert et al. (1990). The solid line is fit of multitarget model to data with par; meters listed in Table 1 and the dashed line is fit of linear repair kinetics model of Wilson et al. (1993). 
the solid line in Figure 2 with the resulting parameters $m=2, D_{0_{M}}=950 \mathrm{~Gy}$. Wilson et al. (1993) formulated a linear kinetics model of repair/misrepair to treat multiple lesion types such as mutation and inactivation and which also considers dose-rate effects. The fit of this model to the X-ray data is shown by the dash line in Figure 2. For our purpose of treating track structure effects on evaluating mutation cross sections, the use of the multitarget model or linear kinetics model for mutations gave similar fits to the data. The multitarget model is used in the figures shown. As described by Wilson et al. (1993), the parameters used by Katz et al (1994) can be used in a linear kinetics model when inteter $m$ values are assumed.

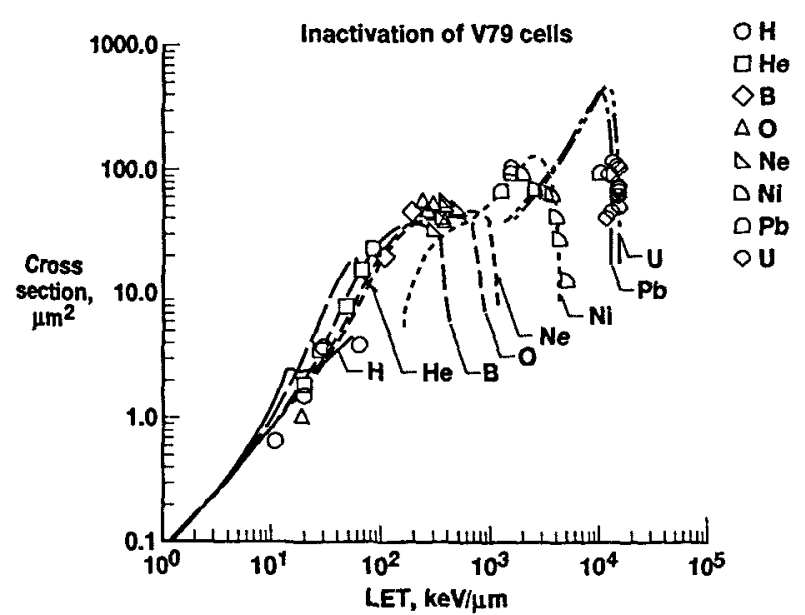

Figure 3. Calculations and experimental values of inactivation cross sections (final slope) for V79 cells plotted versus LET. Data are from Thacker et al. (1979), Kranert, et al. (1990), Belli et al. (1993), and Kiefer et al. (1994).
We have considered only integer $m$ values in our fits, although non-integers would perhaps provide a superior fit. The drop-off in mutation frequency at higher doses of $\mathrm{X}$-rays in Figure 2 is a common feature of such data. The lack of X-ray data at larger doses ( $>10 \mathrm{~Gy}$ ) leaves some uncertainty in the mapping to ion effects using equation 8.

Calculations of inactivation cross sections are compared with experimental data as a function of LET in Figure 3. Shown are the final slope or extrapolated cross sections for several charges. Cellular response parameters for inactivation (Katz et al. 1994) are listed in Table 1. The maximum value of the inactivation cross section versus LET for each specific ion occurs in the energy range of about $5-30 \mathrm{MeV} / \mathrm{u}$. The decrease in the inactivation cross section is called thindown and occurs when $T_{\max }<a_{0_{\mathrm{I}}}$. The nuclear area for V79 cells will vary with cell type, strain, growth conditions, etc., and has been reported as $130 \mu \mathrm{m}^{2}$ by Goodhead et al. (1980) corresponding to the experiments of Thacker et al. (1979), roughly three times the value of $\sigma_{0_{\mathrm{I}}}=42 \cdot 8 \mu \mathrm{m}^{2}$. We note that for a small range of energies and for very large charges, the model inactivation cross sections exceed the reported geometric area of $130 \mu \mathrm{m}^{2}$.

In the evaluation of mutation cross sections using equation 8 the upper limit on $|\vec{r}|$ should be on the order of $1-3 \mu \mathrm{m}$ in view of the measured nuclear area for V79 cells and the fitted values of $\sigma_{0_{1}}$ and $a_{0_{1}}$. We have treated the maximum $r$, denoted $r_{\max }$, as a fitting parameter estimated as $r_{\max }=1.75 \mu \mathrm{m}$ from calculations. We first show plots of $\sigma_{\mathrm{M}}$ for uranium and oxygen ions versus energy in Figure 4 for several fixed values of $r$. For small $r$, virtually no mutations are seen for heavy
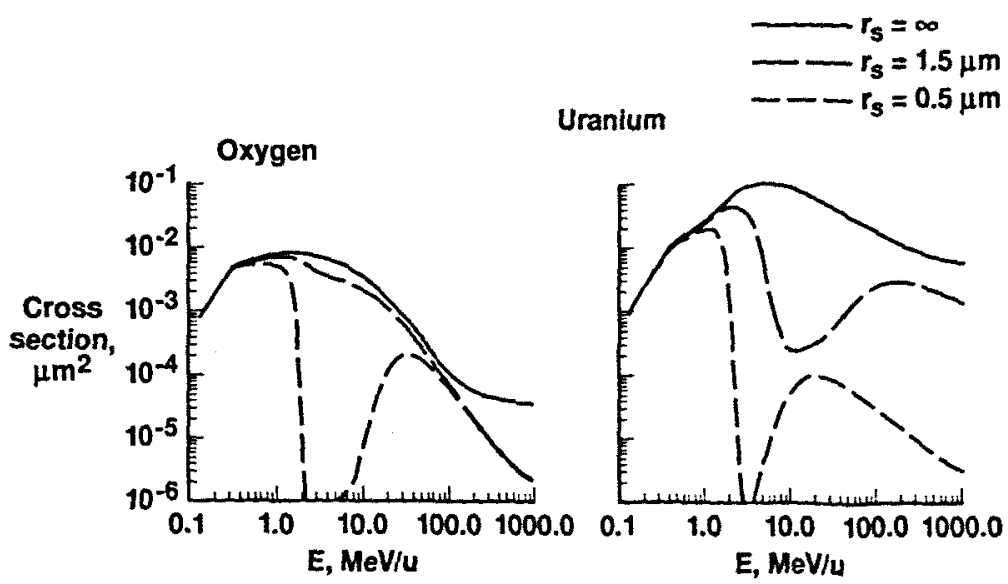

Figure 4. (a) Calculations of mutation cross sections versus energy for oxygen ions at various fixed separation distances from mutation lesion site to inactivation lesion site. (b) Same as for (a) for uranium ions. 


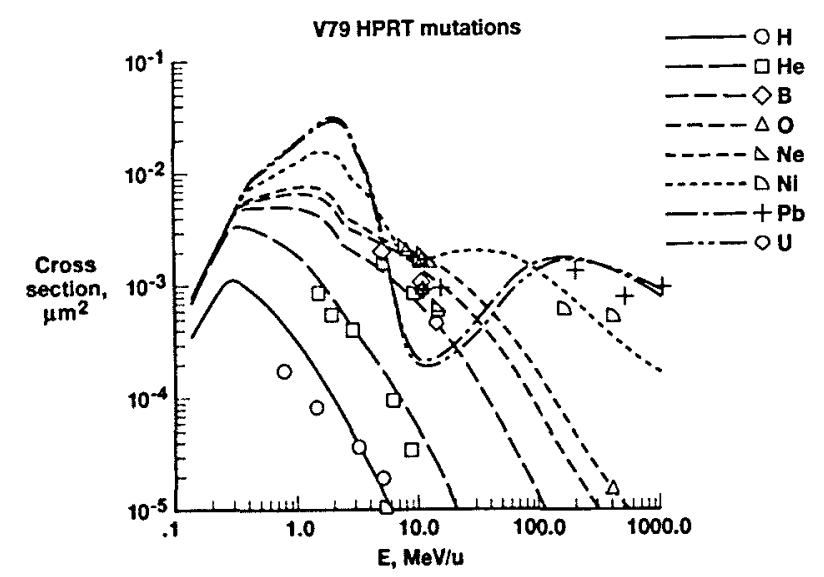

Figure 5. Calculations and experimental values of mutation cross sections plotted versus energy for HPRT mutations in V79 cells. Data are from Thacker et al. (1979), Kranert et al. (1990), Belli et al. (1993), and Kiefer $e t$ al. $(1994)$, and is initial slope data when available.

ions of modest energies where the highest rates of inactivation occur. At low energies $(<1 \mathrm{MeV})$ the mutation cross sections increases where thindown in the inactivation cross sections occur. A second maximum in the inactivation cross section in yeast and bacteria has been observed at low energies (Schneider et al. 1990) which is not accounted for by the $\delta$-ray model and would most likely reduce the mutation cross sections in the energy region from $0 \cdot 1$ to $1.0 \mathrm{MeV} / \mathrm{u}$ over the calculations shown here if the same effect is presented for the inactivation of V79 cells.

In Figure 5 we show the model calculations and experimental values for V79 mutation cross sections versus ion energy for several ion types. The data shown for heavy ions with $Z>8$ are from Kranert et al. (1990) and Kiefer et al. (1994). The data shown for $\mathrm{He}$ and $\mathrm{B}$ are the initial slope cross sections from Thacker t al. (1979) and the data for protons from Belli et al. (1993). Different strains of
V79 cells are used by the authors noted. Cellular response parameters for mutation are listed in Table 2. The value of $a_{0_{\mathrm{M}}}$ most strongly affects the fit for light charged ions and is somewhat sensitive to the angular distribution of secondary electrons assumed in calculations. The $a_{0_{\mathrm{M}}}$ obtained by fits, as noted by Goodhead (1989), corresponds to a large portion of the HPRT gene. The agreement between calculations and experiment is good. Heavy ions are seen to display minima in their mutation capability due to inactivation effects. This minima occurs for kinetic energies from a few to about $30 \mathrm{MeV} / \mathrm{u}$. The overestimation of the model inactivation cross section for uranium in this energy regime affects the mutation cross sections too severely; however, the trends are correct. A statistical test of our fits has not been made at this time. We note that the usefulness of a statistical test of the model may be limited since the measurements discussed are from several laboratories (Thacker et al. 1979, Kranert et al. 1990, Belli et al. 1993). Relativistic heavy ions thus become more effective due to the large radial extent of their tracks from $\delta$-rays. The spreading of the ions track at high energies reduces the effects of inactivation, thus increasing the likelihood of mutation. Note also that ions of moderate charge become more efficient than higher charge ions in the moderate energies region from about 5 to $50 \mathrm{MeV} / \mathrm{u}$. Light ions are only mildly effected by inactivation effects.

In Figure 6 we show the mutation cross sections plotted versus LET. A 'hook' structure distinct from the 'hooks' seen in inactivation cross sections due to thindown effects is observed. A sharp rise in $\sigma_{\mathrm{M}}$ is seen for a narrow band of LETs as correlated with the thindown of $\sigma_{I}$ for the same LET band. A complicated structure of $\sigma_{\mathrm{M}}$ predicted by the model prohibits the use of a single quantity such as LET or $Z^{* 2} / \beta^{2}$ for defining radiation quality as noted by Kranert et al. (1990).

Table 2. Photon initial slopes and geometric parameters for several cell lines

\begin{tabular}{|c|c|c|c|c|}
\hline Cell type & $\begin{array}{l}\text { Initial slope for } \\
\text { HPRT mutations, } \\
\left(10^{7} \mathrm{cG}^{-1}\right)\end{array}$ & $\begin{array}{l}\text { Geometric } \\
\text { area }(\mu \mathrm{m})\end{array}$ & Radius $(\mu \mathrm{m})$ & Reference \\
\hline 179 & $0 \cdot 35$ & 130 & $6 \cdot 4$ & $\begin{array}{l}\text { Thacker et al. (1979) } \\
\text { Goodhead et al. (1980) }\end{array}$ \\
\hline $\begin{array}{l}\text { Human } \\
\text { Iymphoblasts } \\
\text { Human skin }\end{array}$ & $0 \cdot 6$ & 87 & $5 \cdot 3$ & Kronenberg (1994) \\
\hline fibroblasts & $2 \cdot 3$ & 220 & $8 \cdot 4$ & Tsuboi et al. (1992) \\
\hline
\end{tabular}




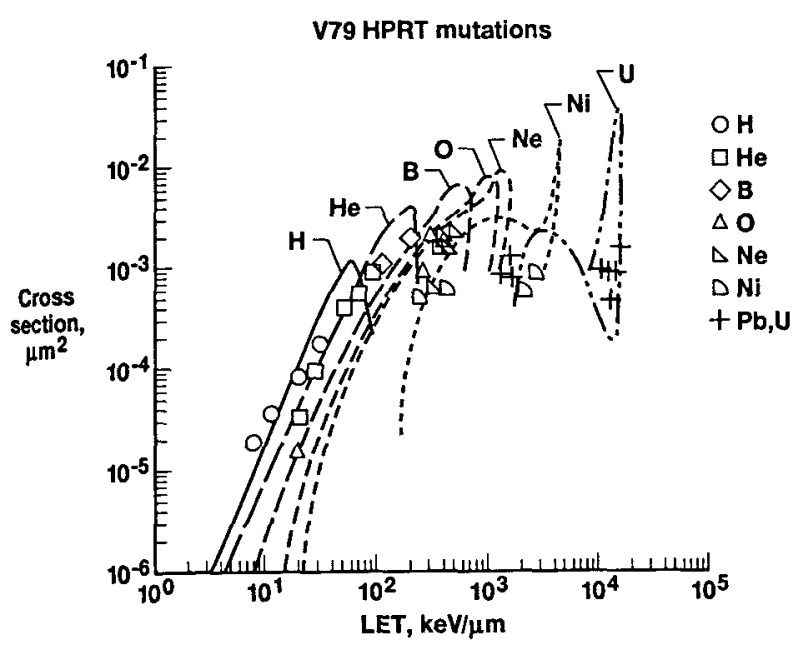

Figure 6. Same as for Figure 5 plotted versus LET.

\section{Discussion and conclusions}

Cross sections for HPRT mutations have also been reported for human fibroblast cells (Cox and Masson 1979, Tsuboi $e t$ al. 1992) and for human Blymphoblastoid cells (Kronenberg et al. 1989, 1994). The fibroblasts' and lymphoblasts' response to heavy ions has been noted to be quite different by Kronenberg (1994) as seen, e.g., in the large differences in cross sections for relativistic iron nuclei of similar energies where the cross sections in fibroblasts are about a factor of 15 larger in the human fibroblasts. The present models suggest that these differences are inherent in the $\gamma$-ray response of individual cell types without regard to any specific expression pathways for heavy ions. In Table 2 we list reported initial slope estimates and cross sectional areas for several cell lines in which HPRT mutations have been measured. We note that the low LET response is different, being larger for the human fibroblasts and similar for the V79 and lymphoblasts. This is also true of the measured cross sectional area with the human fibroblast nuclear area almost twice as large as that of the V79 and lymphoblasts. If the multitarget model or linear kinetics model is fit to the human skin fibroblast data (Tsuboi et al. 1992) with $m=2, D_{0_{\mathrm{M}}}$ of around $650 \mathrm{~Gy}$ is found, which compares with the $950 \mathrm{~Gy}$ found for V79 cells. In contrast with the human lung fibroblast system (Cox and Masson 1979), the human fibroblasts used by Tsuboi et al. (1992) do express curvature in the $\gamma$-ray dose-response for both inactivation and mutation. The linear kinetics model fits both sets of fibroblast lines by introducing a finite misrepair term for the lung fibroblasts. In the cross section formula of equation 8 , the values of $V_{\mathrm{N}}$ are also expected to be increased for the larger nucleus of the human fibroblast cells. It is difficult to determine if an increase in the cell volume will reduce the role of inactivation on the mutation rate. We expect that the differences in low LET response to be sufficient to explain the differences in mutation rates between cell lines without the introduction of any new mechanisms for ions. The recent measurements of Kiefer et al. (1994) using $400 \mathrm{MeV} / \mathrm{u}$ Nickel support this premise where the mutation cross section in V79 cells in $5.0 \times 10^{-4} \mu \mathrm{m}^{2}$ in comparison with $600 \mathrm{MeV} / \mathrm{u}$ iron nuclei in lymphoblasts and fibroblast with mutation cross sections of $3.7 \times 10^{-4}$ and $56.5 \times 10^{-4} \mu \mathrm{m}^{2}$ respectively.

The track structure model uses a model fit to experimental measurements for high dose-rate response to low LET radiations, the radial dose distribution about the path of a heavy ion, and a few geometric parameters to predict the effects of the identical system to an arbitrary ion. In the past this procedure has been shown to be quite successful for describing inactivation cross sections in many biological samples. Herein we have shown that a similar procedure can be applied to predict mutation rates when the effects of inactivation are included by spatially correlating lesion sites. The continued success of the track model in fitting biological data with ion beams suggests that a fundamental approach to biological damage from energetic photons would provide much of the understanding needed for ion beams as well. The action cross sections for mutation plotted versus LET will have a distinct structure due to the effect of inactivation, especially for heavy ions. The use of a single parameter such as LET or $Z^{* 2} / \beta^{2}$ to represent radiation quality is thus even less accurate for mutations than inactivation.

\section{References}

Belu, M., Cera, F., Cherubin, R., Haque, A. M. I., Ianzini, F., Moschin, G., Sapora, O., Simonf, G., Tabocchini, M. A. and Trverton, P., 1993, Inactivation and mutation induction in V79 cells by low energy protons: reevaluation of the results at the LNL facility. International Journal of Radiation Biology, 63, 331-337.

BrAndt, W. and RrTCHIE, R. H., 1974, Primary interactions in the physical stage. In: Physical Mechanisms in Radiation Biology. Edited by: R. D. Cooper and R. W. Wood. (Chicago: Technical Information Services, United States Atomic Energy Commission), pp. 20-50.

Burts, J. J. and KAr, R., 1967, Theory of RBE for heavy ion bombardment of dry enzymes and viruses. Radiation Research, 30, 855-871.

Cox, R. and MASSON, W. K., 1979, Mutation and inactivation of 
cultured mammalian cells exposed to beams of accelerated heavy ions. III. Human dipoid fibroblasts. International Journal of Radiation Biology, 36, 149-160.

Cucinotta, F. A., Katz, R., Wilson, J. W. and Dubey, R. R., 1995b, Heavy Ion Track-Structure Calculations for Radial Dose in Arbitrary Materials, NASA Technical Paper 3497, Washington D.C.

Cucinotta, F. A., Wilson, J. W., Katz, R., Atwell, W., Badwar, G. D. and Shavers, M. R., 1995a, Track structure and radiation transport model for space radiobiology studies. Advances in Space Research. (in press).

Facils, R., Schafer, M., Baltsciutak, K. and Bucker, H., 1983, Inactivation probability of heavy-ion irradiated spores of Bacillus subtilis as a function of radial distance to the particles trajectory. Advances in Space Research, 3, 85-91.

Goonflist, D. T., 1989, Relationships of radiation track structure to biological effect: a re-interpretation of the parameters of the Katz model. Nuclear Tracks and Radiation Measurements, 16, 177-184.

Goonhead, D. T., Munson, R. J., Thacker, J. and Cox, R., 1980, Mutation and inactivation of cultured mammalian cells exposed to beams of accelerated heavy ions. IV. Biophysical interpretation. International Journal of Radiation Biology, 37, 135-167.

Graits, D. (ed), 1973, HZE Particle Effects in Manned Spaceflight. Radiobiology Advisory Panel, Committee on Space Medicine, National Academy of Sciences (Washington: National Academy Press).

Katz, R., ACkerson, B., Homayoonfar, M. and Scharma, S. C., 1971, Inactivation of cells by heavy ion bombardment. Radiation Research, 47, 402-425.

Katz. R. DiNs, D. E. and Sinclair, G. L., 1985, Thindown in radiobiology. Radiation Protection and Dosimetry, 13, 281-284.

Katz, R. Zacharlah, R., Cucinotta, F. A. and Zhang, C., 1994, Survey of radiosensitivity parameters. Radiation Research, 14, 356-365.

Kifser, J.. 1993. Theoretical analysis of heavy ion action on cells: model-free approaches, consequences and radiation protection. In: Biological Effects and Physics of Solar and Galactic Cosmic Radiation. Edited by: C. E. Swenberg, G. Horneck, and E. G. Strassinopoulos Part A (New York: Plenum Press), pp 283-290.

Kiesrr. J. Stou. U. and Schiverder, E., 1994, Mutation induction by heary ions. Adrances in Space Research, 14, (10) $257-265$.

Korminu, F. J and Katz. R., 1968, The energy deposition of clectron beams and delia rays. Physical Reviews, 170, $391-396$.

Korr, M. and Kirfrr, J., 1993, Biological action of single heavy ions on individual yeast cells. In: Biological Effects and
Physics of Solar and Galactic Cosmic Radiation Edited by: C. E. Swenberg, G. Horneck, and E. G. Stassinopoulos, Part A (New York: Plenum Press), pp. 117-124.

Kranert, T., Schneider, E. and Kiefer, J., 1990, Mutation induction in V79 Chinese hamster cells by very heavy ions. International Journal of Radiation Biology, 58, 979-987.

KronenberG, A., 1994, Mutation induction in human lymphoid cells by energetic heavy ions. Advances in Space Research, 14, (10)339-346.

KronenberG, A. and Little, J. B., 1989, Locus specificity for mutation induction in human cells exposed to accelerated heavy ions. International Journal of Radiation Biology, 55, 913-924.

LETT, J. T., Cox, A. E. and STORY, M. D., 1989, The role of repair in the survival of mammalian cells from heavy ion irradiation: approximation to the ideal case of target theory. Advances in Space Research, 9, (10)99-104.

National Council on Radiation Protection Measurements, 1990, The Relative Biological Effectiveness of Radiations of Different Quality. No. 104 (Bethesda, Maryland: NCRP).

Rund, M. E., 1989, User friendly model for the energy distribution of electrons from proton or electron collisons. Nucleic Tracks and Radiation Measurement, 16, 213-222.

Schneiner, E., Kost, M. and Schafer, M., 1990, Inactivation cross sections of yeast and bacteria exposed to heavy ions of low energy $(<600 \mathrm{keV} / \mathrm{u})$. Radiation Protection Dosimal, 31, 291-295.

'Тавата, T., Iто, R. and Oкаве, S., 1972, Generalized semiempirical equations for the extrapolated range of electrons. Nucleic Instruments and Methods, 103, 85-91.

Thacker, J., Stretch, A. and Stephens, M. A., 1979, Mutation and inactivation of cultured mammalian cells exposed to beams of accelerated heavy ions. II. Chinese hamster V79 cells. International Journal of Radiation Biology, 36, $137-248$.

Tond, P. W., 1983, Unique biological aspects of radiation hazards - an overview. Advances in Space Research, 3, $187-192$.

Tsubol, K., Yang, T. C., PARK, M. S. and Chen, D. J., 1992, Cytotic and mutagenic effects of high LET charged particles on human skin fibroblasts. Radiation Research, 129, $171-176$.

Weissbrod, U., Bucker, H., Horneck, G., and Kraft, G., 1992, Heavy-ion effects on bacteria spores: the impact parameter dependence of the inactivation. Radiation Research, 129, 250-257.

Wilson, J. W. CucinotTA, F. A. Shinn, J. L., 1993, Cell kinetics and track structure. In: Biological Effects and Physics of Solar and Galactic Cosmic Radiation. Edited by: C. E. Swenberg, G. Horneck, and E. G. Stassinopoulos, Part A (New York: Plenum Press), pp. 295-338. 